\title{
RAMANUJAN'S ELEMENTARY METHOD IN PARTITION CONGRUENCES
}

\author{
BRUCE C. BERNDT, CHADWICK GUGG, AND SUN KIM
}

\begin{abstract}
Page 182 in Ramanujan's lost notebook corresponds to page 5 of an otherwise lost manuscript of Ramanujan closely related to his paper providing elementary proofs of his partition congruences $p(5 n+4) \equiv 0(\bmod 5)$ and $p(7 n+5) \equiv 0(\bmod 7)$. The claims on page 182 are proved and discussed, and further results depending on Ramanujan's ideas are established.
\end{abstract}

2000 Math. Review Nos. Primary: 11P83; Secondary: 11P81, $05 \mathrm{~A} 17$

Key Words: partitions, congruences, pentagonal number theorem, Jacobi's identity, Winquist's identity.

\section{INTRODUCTION}

Ramanujan published three papers, [12], [13], [14], on congruences for the partition function $p(n)$. However, the second [13] is only a short announcement of results, and the third [14] was extracted by G.H. Hardy from a much longer handwritten manuscript after Ramanujan's death. The latter manuscript, concentrating on both $p(n)$ and Ramanujan's $\tau$-function $\tau(n)$, was published for the first time with Ramanujan's lost notebook [17] in its original handwritten form. Later, this $p(n) / \tau(n)$ manuscript was prepared for journal publication, with amplification of details and extensive commentary, by the first author and K. Ono [7]. That article is reproduced in the book [1] by Andrews and Berndt, with the previous commentary considerably expanded.

Page 182 of [17] is also devoted to the theory of partitions. The number (5) is written in the upper right-hand corner of page 182 in [17], likely indicating that this is the fifth page of a handwritten manuscript. The first and second lines on this page are identical to the second and third lines of equation (11) in [12], [15, p. 211], where Ramanujan begins to relate his elementary proof of $p(5 n+4) \equiv 0(\bmod 5)$. The tagged equation numbers on page 182 are (2.2)-(2.5), which clearly indicate that this page is in Section 2 of this manuscript. However, page 182 is not identical to any page or pages in [12]. Ramanujan's proof of $p(5 n+4) \equiv 0(\bmod 5)$ here is considerably briefer than it is in [12]. Moreover, central to Ramanujan's thoughts is the more general partition function $p_{r}(n)$ defined by

$$
\frac{1}{(q ; q)_{\infty}^{r}}=\sum_{n=0}^{\infty} p_{r}(n) q^{n}, \quad|q|<1,
$$

which is not discussed in [12]. This definition is actually not provided on page 182, but it is clear that it must have been given somewhere in the missing pages 1-4 of the manuscript. Of course, $p_{1}(n)=p(n)$. In a letter to Hardy written from Fitzroy House late in 1918 [8, pp. 192-193], Ramanujan writes, "I have considered more or less exhaustively about the congruency of $p(n)$ and in general that of $p_{r}(n)$ where

$$
\sum p_{r}(n) x^{n}=\frac{1}{(x ; x)_{\infty}^{r}},
$$


by four different methods." This declaration appears to imply that he had established several results about $p_{r}(n)$, which quite likely were discussed in the manuscript for which we now unfortunately have only page 5 .

Ramanujan deduces the congruence $p(5 n-1) \equiv 0(\bmod 5)$ from the congruence $p_{-4}(5 n-1) \equiv 0(\bmod 5)$, just as he does in $[12]$ without using this notation. Ramanujan then remarks that, "Precisely in the same way we can show that

$$
p_{-4}\left(n \varpi-\frac{\varpi+1}{6}\right) \equiv 0(\bmod \varpi)
$$

where $\varpi$ is a prime of the form $6 \lambda-1 \ldots$ "He then states a more general theorem. It is therefore quite clear that Ramanujan's paper [12] was likely extracted from a greatly expanded longer manuscript, which was not a part of his $p(n) / \tau(n)$ manuscript and which, except for this single page, has been lost.

After Ramanujan, the function $p_{r}(n)$, in another notation $p_{-r}(n)$, was studied by, in particular, M. Newman [9], K.G. Ramanathan [10], and A.O.L. Atkin [3], who were also interested in its congruences. However, they confined themselves to congruences satisfied by a small set of primes and powers thereof, in contrast to Ramanujan's theorems on page 182 satisfying an infinite set of primes.

The keys to Ramanujan's elementary methods are the pentagonal number theorem

$$
\sum_{n=-\infty}^{\infty}(-1)^{n} q^{n(3 n-1) / 2}=(q ; q)_{\infty}
$$

and Jacobi's identity

$$
\sum_{n=0}^{\infty}(-1)^{n}(2 n+1) q^{n(n+1) / 2}=(q ; q)_{\infty}^{3} .
$$

It is well known that no one has been able to employ these identities to give an elementary proof of Ramanujan's congruence $p(11 n+6) \equiv 0(\bmod 11)$. The only known elementary proof of this congruence is due to L. Winquist [18] and employs an identity that is now known by his name. At the end of Section 2, for the first time, we utilize Winquist's identity to derive new congruences for special instances of $p_{r}(n)$.

Ramanujan's elementary methods focusing on Jacobi's identity have been utilized and generalized by several authors. The most extensive applications of this method have been made by G.E. Andrews and R. Roy [2]; their paper contains several additional references. There are two further identities in the spirit of Jacobi's identity, both due to Ramanujan [16], and we employ them in Section 3 to derive two analogues of the general theorem of Andrews and Roy.

\section{Page 182 in Ramanujan's Lost Notebook}

A brief account of page 182 along with a proof of the first entry below has been given by Ramanathan [11]. 
Entry 2.1 (p. 182). Let $\delta$ denote any integer, and let $n$ denote a nonnegative integer. Suppose that $\varpi$ is a prime of the form $6 \lambda-1$. Then

$$
p_{\delta \varpi-4}\left(n \varpi-\frac{\varpi+1}{6}\right) \equiv 0(\bmod \varpi) .
$$

Proof. Consider

$$
\begin{aligned}
& \sum_{n=0}^{\infty} p_{\delta \varpi-4}(n) q^{n+\lambda}=(q ; q)_{\infty}^{-\delta \varpi}(q ; q)_{\infty}^{3}(q ; q)_{\infty} q^{\lambda} \\
& \equiv\left(q^{\varpi} ; q^{\varpi}\right)_{\infty}^{-\delta} \sum_{\mu=0}^{\infty} \sum_{\nu=-\infty}^{\infty}(-1)^{\mu+\nu}(2 \mu+1) q^{\frac{1}{2} \mu(\mu+1)+\frac{1}{2} \nu(3 \nu+1)+\lambda}(\bmod \varpi),
\end{aligned}
$$

upon the use of Euler's pentagonal number theorem (1.2) and Jacobi's identity (1.3). We want to examine those terms for which

$$
\frac{1}{2} \mu(\mu+1)+\frac{1}{2} \nu(3 \nu+1)+\frac{\varpi+1}{6} \equiv 0(\bmod \varpi) .
$$

Our goal is to prove that

$$
\varpi \mid(2 \mu+1) .
$$

Multiply (2.3) by 24 to obtain the equivalent congruence

$$
12 \mu(\mu+1)+12 \nu(3 \nu+1)+4 \varpi+4 \equiv 0(\bmod \varpi)
$$

or

$$
3(2 \mu+1)^{2}+(6 \nu+1)^{2} \equiv 0(\bmod \varpi)
$$

Using the fact that, for each prime $p$, the Legendre symbol $\left(\frac{-1}{p}\right)=(-1)^{(p-1) / 2}$, and the law of quadratic reciprocity, we find that

$$
\left(\frac{-3}{\varpi}\right)=\left(\frac{\varpi}{3}\right)=\left(\frac{-1}{3}\right)=-1 .
$$

Thus, the only way that (2.5) can hold is for (2.4) to happen. But then, from the right-hand side of (2.2), we can conclude that

$$
p_{\delta \varpi-4}\left(n \varpi-\frac{\varpi+1}{6}\right) \equiv 0(\bmod \varpi) .
$$

Thus, the proof is complete.

Corollary 2.2 (p. 182). For each positive integer $n$,

$$
\begin{aligned}
p_{6}(5 n-1) & \equiv 0(\bmod 5), \\
p_{7}(11 n-2) & \equiv 0(\bmod 11) .
\end{aligned}
$$

Proof. The first congruence arises from the case $\varpi=5$ and $\delta=2$, while the second arises from the case $\varpi=11$ and $\delta=1$ in Entry 2.1. 
Next, Ramanujan gives an elementary proof of the congruence $p(7 n-2) \equiv 0(\bmod 7)$. He begins with the same first three lines of [12, eq. (13)], [15, p. 212], and then argues in a somewhat more abbreviated fashion than he does in [12] to deduce the congruence

$$
p_{-6}(7 n-2) \equiv 0(\bmod 49) \text {, }
$$

from which it follows that

$$
p(7 n-2) \equiv 0(\bmod 7) .
$$

It should be remarked that the stronger congruence (2.6) is not mentioned by Ramanujan in [12], although it is implicit in his argument.

Unfortunately, the one-page manuscript ends with (2.7). It would seem that Ramanujan would have next offered a theorem analogous to Entry 2.1, and so we shall state and prove such a theorem here, but, of course, Ramanujan probably would have had lots more to say to us, if his manuscript had survived.

Theorem 2.3. For a prime $\varpi$ with $4 \mid(\varpi+1)$, any integer $\delta$, and any positive integer $n$,

$$
p_{\delta \varpi-6}\left(n \varpi-\frac{\varpi+1}{4}\right) \equiv 0(\bmod \varpi) .
$$

In the case $\delta=0$ above, we can strengthen (2.8).

Entry 2.4 (p. 182). We have

$$
p_{-6}\left(n \varpi-\frac{\varpi+1}{4}\right) \equiv 0\left(\bmod \varpi^{2}\right) .
$$

Observe that (2.6) is the special case $\varpi=7$ of (2.9), and so, with slight exaggeration, we affixed "p. 182" to the entry above.

Corollary 2.5. For each positive integer n,

$$
p_{3 \delta-6}(3 n-1) \equiv 0(\bmod 3) .
$$

Proof. Set $\varpi=3$ in Theorem 2.3.

For the case $\delta=3$ in (2.10), N.D. Baruah and K.K. Ojah [4], using more sophisticated means, obtained the stronger result

$$
p_{3}(3 n-1) \equiv 0\left(\bmod 3^{2}\right) .
$$

Proof of Theorem 2.3. Consider, for $\lambda=(\varpi+1) / 4$,

$$
\begin{aligned}
& \sum_{n=0}^{\infty} p_{\delta \varpi-6}(n) q^{n+\lambda}=(q ; q)_{\infty}^{-\delta \varpi}(q ; q)_{\infty}^{6} q^{\lambda} \\
& \equiv\left(q^{\varpi} ; q^{\varpi}\right)_{\infty}^{-\delta} \sum_{\mu=0}^{\infty} \sum_{\nu=0}^{\infty}(-1)^{\mu+\nu}(2 \mu+1)(2 \nu+1) q^{\frac{1}{2} \mu(\mu+1)+\frac{1}{2} \nu(\nu+1)+\lambda}(\bmod \varpi),
\end{aligned}
$$

upon the use of Jacobi's identity (1.3). We need to show that if

$$
\frac{1}{2} \mu(\mu+1)+\frac{1}{2} \nu(\nu+1)+\frac{\varpi+1}{4} \equiv 0(\bmod \varpi),
$$

then

$$
\varpi^{2} \mid(2 \mu+1)(2 \nu+1)
$$


The congruence (2.8) will then follow from (2.13) and (2.11). Multiply (2.12) by 8 to obtain

$$
4 \mu(\mu+1)+4 \nu(\nu+1)+2 \varpi+2 \equiv 0(\bmod \varpi)
$$

or

$$
(2 \mu+1)^{2}+(2 \nu+1)^{2} \equiv 0(\bmod \varpi)
$$

Since

we conclude that

$$
\left(\frac{-1}{\varpi}\right)=-1
$$

$$
\varpi \mid(2 \mu+1) \text { and } \varpi \mid(2 \nu+1)
$$

which completes the proof of (2.13).

Observe that if $\delta=0$, then the congruence in (2.11) can be replaced by an equality. Hence, in (2.8), the congruence modulo $\varpi$ can be replaced by a congruence modulo $\varpi^{2}$ in view of (2.13). Entry 2.4 therefore follows.

Although Entry 2.1 and Theorem 2.3 are not special cases of the general theorem of Andrews and Roy [2], they would be instances of the general theorem envisioned by the authors in Section 5 of their paper [2].

Recall next that a corollary of Winquist's identity is given by [18]

$48(q ; q)_{\infty}^{10}=\sum_{m, n=-\infty}^{\infty}(-1)^{m+n}\left((6 m+3)^{3}(6 n+1)-(6 m+3)(6 n+1)^{3}\right) q^{\frac{1}{2}\left(3 m^{2}+3 m+3 n^{2}+n\right)}$.

Theorem 2.6. For a prime $\varpi$ with $12 \mid(\varpi+1)$, and any integer $\delta$, we have

$$
p_{\delta \varpi-10}\left(n \varpi-\frac{5(\varpi+1)}{12}\right) \equiv 0(\bmod \varpi) .
$$

Proof. Let $\lambda=5(\varpi+1) / 12$, and from (2.14) consider

$$
\begin{aligned}
& \sum_{n=0}^{\infty} p_{\delta \varpi-10}(n) q^{n+\lambda}=(q ; q)_{\infty}^{-\delta \varpi}(q ; q)_{\infty}^{10} q^{\lambda} \\
& \equiv\left(q^{\varpi} ; q^{\varpi}\right)_{\infty}^{-\delta} \frac{1}{48} \sum_{m, n=-\infty}^{\infty}(-1)^{m+n}\left((6 m+3)^{3}(6 n+1)\right. \\
& \\
&\left.-(6 m+3)(6 n+1)^{3}\right) q^{\frac{1}{2}\left(3 m^{2}+3 m+3 n^{2}+n\right)+\lambda}(\bmod \varpi) .
\end{aligned}
$$

If

$$
\frac{1}{2}\left(3 m^{2}+3 m+3 n^{2}+n\right)+\lambda \equiv 0(\bmod \varpi),
$$

then upon multiplying both sides above by 24 , we find that

$$
12\left(3 m^{2}+3 m+3 n^{2}+n\right)+10(\varpi+1) \equiv 0(\bmod \varpi),
$$

or

$$
(6 m+3)^{2}+(6 n+1)^{2} \equiv 0(\bmod \varpi) .
$$


Since

$$
\left(\frac{-1}{\varpi}\right)=-1
$$

we see that

$$
\varpi \mid(6 m+3) \quad \text { and } \quad \varpi \mid(6 n+1) .
$$

Using these observations in (2.15), we complete the proof.

We observe that in the special case $\delta=0$, our proof yields a stronger result.

Corollary 2.7. For a prime $\varpi$ with $12 \mid(\varpi+1)$, we have

$$
p_{-10}\left(n \varpi-\frac{5(\varpi+1)}{12}\right) \equiv 0\left(\bmod \varpi^{4}\right) .
$$

\section{Two Further General Congruences}

Our analogues of the main theorem of Andrews and Roy [2] are dependent on the two identities

$$
\sum_{j=-\infty}^{\infty}(6 j+1) q^{3 j^{2}+j}=\left(q^{2} ; q^{2}\right)_{\infty}^{3}\left(q^{2} ; q^{4}\right)_{\infty}^{2}
$$

and

$$
\sum_{j=-\infty}^{\infty}(3 j+1) q^{3 j^{2}+2 j}=\left(q^{2} ; q^{2}\right)_{\infty}\left(q ; q^{2}\right)_{\infty}^{2}\left(q^{4} ; q^{4}\right)_{\infty}^{2}
$$

which arise from the quintuple product identity and which are found as Entries 8(ix), (x) in Chapter 17 of Ramanujan's second notebook [16]. For proofs, see [5, pp. 118-119] or [6, pp. 20-22]. More historical information can be found in [6, p. 25].

For each integer $m$, we shall denote by $\bar{m}$ the multiplicative inverse of $m(\bmod p)$. We prove the following theorems.

Theorem 3.1. Suppose that $p$ is a prime $>3,0<a<p$, and $a$ and $b$ are integers. Assume that $-6 a$ is a quadratic non-residue modulo $p$. Suppose that $\left\{\alpha_{n}\right\}_{n=-\infty}^{\infty}=$ $\left\{\alpha_{n}\left(z_{1}, z_{2}, \ldots, z_{j}\right)\right\}$ is a doubly infinite sequence of Laurent polynomials over $\mathbb{Z}$ with variables $z_{1}, \ldots, z_{j}$ independent of $q$. Then there is an integer $c$ such that the coefficients of $z_{1}^{m_{1}} z_{2}^{m_{2}} \cdots z_{j}^{m_{j}} q^{p N}$ in

$$
\frac{q^{c} \sum_{n=-\infty}^{\infty} \alpha_{n} q^{a\left(n^{2}-n\right) / 2+b n}}{\left(q^{2} ; q^{2}\right)_{\infty}^{p-3}\left(q^{2} ; q^{4}\right)_{\infty}^{p-2}}
$$

are divisible by $p$. The integer $c=c_{p}(a, b)$ may be chosen as the least positive integer congruent to $\overline{24}\left(3 a(2 b \bar{a}-1)^{2}+2\right)(\bmod p)$. 
Proof. By (3.1) and the hypotheses of the theorem, we note that

$$
\begin{aligned}
& \frac{q^{c} \sum_{n=-\infty}^{\infty} \alpha_{n} q^{a\left(n^{2}-n\right) / 2+b n}}{\left(q^{2} ; q^{2}\right)_{\infty}^{p-3}\left(q^{2} ; q^{4}\right)_{\infty}^{p-2}} \\
& =\frac{q^{c} \sum_{n=-\infty}^{\infty} \sum_{j=-\infty}^{\infty}(6 j+1) \alpha_{n} q^{a\left(n^{2}-n\right) / 2+b n+3 j^{2}+j}}{\left(q^{2} ; q^{2}\right)_{\infty}^{p}\left(q^{2} ; q^{4}\right)_{\infty}^{p}} \\
& \equiv \frac{q^{c} \sum_{n=-\infty}^{\infty} \sum_{j=-\infty}^{\infty}(6 j+1) \alpha_{n} q^{a\left(n^{2}-n\right) / 2+b n+3 j^{2}+j}}{\left(q^{2 p} ; q^{2 p}\right)_{\infty}\left(q^{2 p} ; q^{4 p}\right)_{\infty}}(\bmod p) .
\end{aligned}
$$

In the last expression, we see that the denominator is a function of $q^{p}$. Let us now examine the exponent of $q$ in the numerator; for ease of computation, we multiply by 24 to achieve

$$
\begin{aligned}
& 24 c+3 a\left(4 n^{2}-4 n\right)+24 b n+72 j^{2}+24 j \\
& \equiv 3 a(2 n+2 b \bar{a}-1)^{2}+2(6 j+1)^{2}(\bmod p) .
\end{aligned}
$$

Now we observe that if $j \equiv(p-1) / 6(\bmod p)$ (i.e., $(6 j+1) \equiv 0(\bmod p)$,), then the last expression above is congruent to $0(\bmod p)$ precisely when

$$
n \equiv(1-2 b \bar{a}) \overline{2} \equiv \frac{p+1}{2}-b \bar{a}(\bmod p) .
$$

If $j \not \equiv(p-1) / 6(\bmod p)$, then the last expression in $(3.5)$ can never be congruent to $0(\bmod p)$, because, by the assumption that $-6 a$ is a quadratic non-residue modulo $p$, exactly one of

$$
-3 a(2 n+2 b \bar{a}-1)^{2} \text { and } 2(6 j+1)^{2}
$$

is a quadratic residue modulo $p$, and so they cannot be congruent to each other modulo $p$. Therefore the coefficients of $q^{p N}$ in (3.4) are all linear combinations over $p \mathbb{Z}$ of various $\alpha_{n}$. This completes the proof.

Similarly, from (3.2), we can derive the next theorem. We forego the proof.

Theorem 3.2. Suppose that $p$ is a prime $>3,0<a<p$, and $a$ and $b$ are integers. Let -6 a be a quadratic non-residue modulo p. Suppose $\left\{\alpha_{n}\right\}_{n=-\infty}^{\infty}=\left\{\alpha_{n}\left(z_{1}, z_{2}, \ldots, z_{j}\right)\right\}$ is a doubly infinite sequence of Laurent polynomials over $\mathbb{Z}$ with variables $z_{1}, \ldots, z_{j}$ independent of $q$. Then there exists an integer $c$ such that the coefficients of $z_{1}^{m_{1}} z_{2}^{m_{2}} \cdots z_{j}^{m_{j}} q^{p^{N}}$ in

$$
\frac{q^{c} \sum_{n=-\infty}^{\infty} \alpha_{n} q^{a\left(n^{2}-n\right) / 2+b n}}{\left(q^{2} ; q^{2}\right)_{\infty}^{p-1}\left(q ; q^{2}\right)_{\infty}^{p-2}\left(q^{4} ; q^{4}\right)_{\infty}^{p-2}}
$$

are divisible by $p$. The integer $c=c_{p}(a, b)$ may be chosen as the least positive integer congruent to $\overline{24}\left(3 a(2 b \bar{a}-1)^{2}+8\right)(\bmod p)$.

Applications analogous to those made by Andrews and Roy in Section 4 of their paper [2] can be made here, but since they are easily deduced and no new ideas are involved, we do not proceed any further. 


\section{REFERENCES}

[1] G.E. Andrews and B.C. Berndt, Ramanujan's Lost Notebook, Part III, Springer, New York, to appear.

[2] G.E. Andrews and R. Roy, Ramanujan's method in q-series congruences, Elec. J. Combin. 4(2) (1997), R2, 7pp.

[3] A.O.L. Atkin, Ramanujan congruences for $p_{k}(n)$, Canad. J. Math. 20 (1968), 67-78.

[4] N.D. Baruah and K.K. Ojah, Some congruences deducible from Ramanujan's cubic continued fraction, Internat. J. Number Thy., to appear.

[5] B.C. Berndt, Ramanujan's Notebooks, Part III, Springer-Verlag, New York, 1991.

[6] B.C. Berndt, Number Theory in the Spirit of Ramanujan, American Mathematical Society, Providence, RI, 2006.

[7] B.C. Berndt and K. Ono, Ramanujan's unpublished manuscript on the partition and tau functions with proofs and commentary, Sém. Lotharingien de Combinatoire 42 (1999), 63 pp.; in The Andrews Festschrift, D. Foata and G.-N. Han, eds., Springer-Verlag, Berlin, 2001, pp. 39-110.

[8] B.C. Berndt and R.A. Rankin, Ramanujan: Letters and Commentary, American Mathematical Society, Providence, RI, 1995; London Mathematical Society, London, 1995.

[9] M. Newman, Congruences for the coefficients of modular forms and some new congruences for the partition function, Canad. J. Math. 9 (1957), 549-552.

[10] K.G. Ramanathan, Identities and congruences of the Ramanujan type, Canad. J. Math. 2 (1950), $168-178$.

[11] K.G. Ramanathan, Ramanujan and the congruence properties of partitions, Proc. Indian Acad. Sci. (Math. Sci.) 89 (1980), 133-157.

[12] S. Ramanujan, Some properties of $p(n)$, the number of partitions of $n$, Proc. Cambridge Philos. Soc. 19 (1919), 207-210.

[13] S. Ramanujan, Congruence properties of partitions, Proc. London Math. Soc. 18 (1920), xix.

[14] S. Ramanujan, Congruence properties of partitions, Math. Z. 9 (1921), 147-153.

[15] S. Ramanujan, Collected Papers, Cambridge University Press, Cambridge, 1927; reprinted by Chelsea, New York, 1962; reprinted by the American Mathematical Society, Providence, RI, 2000.

[16] S. Ramanujan, Notebooks (2 volumes), Tata Institute of Fundamental Research, Bombay, 1957.

[17] S. Ramanujan, The Lost Notebook and Other Unpublished Papers, Narosa, New Delhi, 1988.

[18] L. Winquist, An elementary proof of $p(11 n+6) \equiv 0(\bmod 11)$, J. Comb. Thy. 6 (1969), 56-59.

Department of Mathematics, University of Illinois, 1409 West Green Street, UrBANA, IL 61801, USA

E-mail address: berndt@illinois.edu

Department of Mathematics, Georgia Southwestern State University, 800 Georgia Southwestern State University Drive, Americus, GA 31709, USA

E-mail address: cgugg@canes.gsw.edu

Department of Mathematics, Pennsylvania State University, University Park, PA 16802, USA

E-mail address: kim@math.psu.edu 